\title{
O JOGO COMO FERRAMENTA DE ENSINO E APRENDIZAGEM EM FONOAUDIOLOGIA ${ }^{1}$
}

\author{
EL JUEGO COMO UNA HERRAMIENTA DE ENSEÑANZA Y APRENDIZAJE \\ EN LA TERAPIA DEL HABLA
}

\section{GAMING AS A TEACHING AND LEARNING TOOL IN SPEECH, LANGUAGE AND HEARING}

\author{
Kelly da SILVA ${ }^{2}$ \\ Raphaela Barroso GUEDES-GRANZOTTI ${ }^{3}$ \\ Carla Patrícia Hernandez Alves Ribeiro CÉSAR ${ }^{4}$ \\ Rodrigo DORNELAS ${ }^{5}$
}

RESUMO: Objetivo: descrever a percepção de discentes sobre a utilização da estratégia do jogo detetive em metodologias ativas para revisão de conteúdo. Método: 22 discentes de ambos os sexos e com média de 23 anos participaram de um jogo de tabuleiro como recurso didático-pedagógico para revisão de conteúdo de um módulo teórico-prático de um curso de graduação em Fonoaudiologia, sobre o tema Linguagem no adulto e idoso. Questões conceituais e casos clínicos foram apresentados para a resolução dos participantes, para que pudessem integrar a teoria com a prática clínica. Os participantes responderam a uma questão norteadora sobre a dinâmica proposta e os resultados foram tratados com técnica de Análise de Conteúdo. Resultados: a estratégia foi descrita como uma intervenção dinâmica, que se opõe ao modelo não participativo, sendo considerada como de eficiência consistente e significativa para a revisão do conteúdo almejado, como favorecedora para a construção do conhecimento coletivo e adequado ao propósito do módulo. Conclusão: as respostas obtidas evidenciaram a percepção positiva dos discentes da pesquisa quanto à utilização do jogo "Detetive da Linguagem" como método de ensino e aprendizagem.

PALAVRAS-CHAVE: Materiais de ensino. Jogos experimentais. Fonoaudiologia.

RESUMEN: Objetivo: describir la percepción de los estudiantes sobre el uso de la estrategia del juego de detectives en metodologías activas para la revisión de contenidos. Método: 22 estudiantes de ambos sexos y con un promedio de 23 años

\footnotetext{
${ }^{1}$ Trabalho realizado no Departamento de Fonoaudiologia da Universidade Federal de Sergipe-UFS, Campus Prof. Antônio Garcia Filho, Lagarto/SE, Brasil. Conflitos de interesse: Nada a declarar. Fonte de Financiamento: Inexistente. Comitê de Ética em Pesquisa sob o número CAAE 33665414.6.0000.5546.

${ }^{2}$ Universidade Federal de Sergipe (Ufs), Lagarto - SE - Brasil. Professora Doutora do Departamento de Fonoaudiologia. E-mail: kelly_fonousp@yahoo.com.br.

${ }^{3}$ Universidade Federal de Sergipe (Ufs), Lagarto - SE - Brasil. Professora Doutora do Departamento de Fonoaudiologia. E-mail: raphaelabgg@gmail.com.

${ }^{4}$ Universidade Federal de Sergipe (Ufs), Lagarto - SE - Brasil. Professora Doutora do Departamento de Fonoaudiologia. E-mail: carlacesar@globo.com.

${ }^{5}$ Universidade Federal de Sergipe (Ufs), Lagarto - SE - Brasil. Professor Doutor do Departamento de Fonoaudiologia. E-mail: rodrigodornela@uol.com.br
} 
participaron en un juego de mesa como recurso didáctico-pedagógico para la revisión del contenido de un módulo teórico-práctico de un curso de posgrado en logopedia, sobre el tema de la lengua en el adulto y Viejo. Las preguntas conceptuales y los casos clínicos fueron presentados para la resolución de los participantes, de modo que pudieran integrar la teoría con la práctica clínica. Los participantes respondieron a una pregunta orientadora sobre la dinámica propuesta y los resultados se trataron con la técnica de análisis de contenido. Resultados: la estrategia se describió como una intervención dinámica, que se opone al modelo no participativo, siendo considerada de una eficacia consistente y significativa para la revisión del contenido deseado, como facilitador para la construcción de la Conocimiento colectivo y adecuado a los efectos del módulo. CONCLUSIÓN: las respuestas obtenidas destacaron la percepción positiva de la investigación de los estudiantes sobre el uso del juego "detective de la lengua" como método de enseñanza y aprendizaje.

PALABRAS CLAVE: Materiales didácticos. Juegos experimentales. Discurso.

ABSTRACT: Objective: To describe the perception of students about the use of the detective game strategy in active methodologies for content review. Method: 22 students of both sexes, with an average of 23 years old participated in a board game as a didactic-pedagogic resource for content of a theoretical and practical module for the review of an undergraduate course in Speech, Language and Hearing Sciences on the topic Language in adults and elderly. Conceptual issues and clinical cases were presented to the resolution of the participants, so that, they could integrate theory with clinical practice. The participants responded to a guiding question on the proposed dynamics and the results were handled with content analysis technique. Results: the strategy was described as a dynamic intervention, which opposes the non-participatory model, considered with consistent and significant efficiency to the review of the desired content as flattering to the construction of collective and appropriate knowledge to the module's purpose. Conclusion: the responses obtained showed a positive perception of the students of the research regarding the use of the "Language Detective" game as a teaching and learning method.

KEYWORDS: Teaching Materials. Games Experimental. Speech, Language and Hearing Sciences.

\section{Introdução}

Atualmente, um grande desafio aos educadores do ensino superior é propiciar uma aprendizagem significativa e efetiva aos discentes, auxiliando no desenvolvimento de habilidades e competências necessárias para a sua prática profissional. Neste caminho, diversas formas de metodologias ativas têm sido utilizadas, tendo como principal objetivo colocar o discente no centro deste processo de aprendizagem. Para isso é de suma importância diversificar os métodos de ensino e os recursos pedagógicos (ZUANON; DINIZ; NASCIMENTO, 2010). 
Transcender a linha que separa a teoria da prática clínica é uma necessidade e um desafio e, por este motivo, ferramentas pedagógicas que facilitem esta aproximação ganham destaque por interligar o cotidiano da prática profissional às teorias e técnicas utilizadas na área da saúde (JUSTUS; FRANCISCO, 2012). Dentre as possibilidades de ferramentas que podem ser utilizadas é possível citar os jogos como recurso facilitador na construção do conhecimento, por propiciar motivação, raciocínio, argumentação e a interação entre docentes e discentes (LEGEY, 2012).

Como descrito por Coscrato, Pina e Melo (2010), os jogos de cartas e tabuleiros, à medida que proporcionam a mediação da aprendizagem, estimulam a compreensão do assunto a ser aprendido de forma prazerosa, por meio da reflexão sobre o conhecimento adquirido e a formação de relações entre o conhecimento proporcionado pelo lúdico e a realidade vivenciada, que engloba os aspectos comportamentais individuais e coletivos.

Fonseca; Scochi e Mello (2002) realizaram um jogo de tabuleiro com 16 puérperas e dois pais sobre aleitamento materno e cuidados com recém-nascidos, sendo possível constatar que a estratégia surtiu efeitos benéficos entre os envolvidos, facilitando a troca de experiências entre as puérperas e o desenvolvimento da educação em saúde, como desejado.

Repensar a prática e transformá-la é uma das premissas de toda metodologia de ensino, fazendo com que todos os envolvidos no processo de ensino e aprendizagem aprendam juntos e reformulem os seus conceitos, de forma ativa durante todo o percurso de construção da aprendizagem (Dornelas et al., 2016).

Na direção de integrar teoria e prática de forma significativa, foi criado em 2011 o curso de Fonoaudiologia em uma Universidade Federal, que tem como proposta pedagógica o uso exclusivo de Metodologias Ativas de ensino, sendo o primeiro no país com esse perfil (DORNELAS et al, 2014).

A integralização do currículo é realizada em quatro anos, ou quatro Ciclos, sendo cada Ciclo constituído de Módulos teóricos e práticos que têm objetivo de analisar os pressupostos teórico-práticos da Fonoaudiologia dentro de eixos temáticos estabelecidos no ementário do projeto pedagógico, levando os discentes a conciliar a parte teórica, ofertada de forma paralela na forma de Aprendizagem Baseada em Problemas (ABP), com as habilidades e competências necessárias para a prática clínica futura (DORNELAS et al, 2014; GUEDES-GRANZOTTI et al, 2015).

Para isso, diversas estratégias pedagógicas têm sido idealizadas pelos docentes para fomentar o raciocínio clínico e as tomadas de decisões, mediante: casos clínicos 
simulados, teatro, criação de cordel, paródias musicais, simulação de atendimento e jogos de tabuleiro. Desta forma, o objetivo deste estudo foi descrever a percepção de discentes sobre a utilização da estratégia, em metodologias ativas, do jogo detetive para revisão de conteúdo.

\section{Método}

Esta pesquisa foi aprovada pelo Comitê de Ética em Pesquisa sob o número CAAE 33665414.6.0000.5546. de acordo com os preceitos estabelecidos pela Resolução 466/2012.

Os sujeitos da pesquisa foram 22 discentes de um Curso de Fonoaudiologia em uma Universidade Federal, matriculados, durante o ano letivo de 2015, no módulo teórico-prático "Práticas Clínicas em Fonoaudiologia - módulo de linguagem em adultos e idosos", que ocorre no terceiro ciclo do curso, e que aceitaram participar da pesquisa assinando o Termo de Consentimento Livre e Esclarecido. A atividade foi realizada no penúltimo encontro do referido módulo, em três turmas diferentes, sendo uma turma com oito e duas turmas com sete discentes.

O jogo, utilizado como estratégia pedagógica, foi denominado como "Detetive de Linguagem", sendo esta uma atividade de tabuleiro, confeccionado em madeira e coberto com Espuma Vinílica Acetinada (EVA), idealizado pela docente responsável; o vencedor do jogo é o grupo que, ao chegar a final, obter a maior pontuação. Algumas "casas" do tabuleiro apresentam perguntas referentes a neuroanatomia, conceitos teóricos e aspectos clínicos das alterações de linguagem no adulto e no idoso, e, em outras "casas" com o dizer CARTÃO, o discente é levado a retirar uma carta com dicas para elucidação de um caso clínico.

Antes de iniciar o jogo, os discentes se dividem em três grupos, por afinidade, e cada grupo escolhe uma cor entre verde, azul ou vermelha, sendo que cada cor corresponde a um diferente caso clínico. Cada grupo, em sua vez, deverá jogar o dado, e andar com seu pino o número de casas indicado; caso caia em uma "casa" com uma pergunta deverá respondê-la, se acertar ganha um ponto e se errar o grupo à sua direita terá a chance de responder. Caso caia na casa CARTÃO, o grupo deve retirar uma carta correspondente à cor escolhida com informações referentes à identificação do paciente, dados da anamnese e da avaliação fonoaudiológica, que irão possibilitar a elucidação do caso clínico. 
Ao final do jogo, os grupos devem identificar o provável diagnóstico fonoaudiológico e médico. Caso a resposta esteja correta, o grupo ganha cinco pontos que irão se somar aos pontos obtidos com as respostas corretas no decorrer do percurso. Após esta fase, foi solicitado aos discentes que elaborassem o planejamento terapêutico do caso clínico para apresentar no próximo encontro.

O jogo teve duração aproximada de três horas; ao final, os participantes tiveram uma hora para responder, em uma folha sulfite, a seguinte questão norteadora: "Em sua opinião, como foi o uso do jogo detetive como estratégia de revisão de conteúdo?”, para que o sujeito participante pudesse expor, individualmente, sua percepção sobre a utilização do jogo "Detetive de Linguagem" como estratégia de revisão de conteúdo do módulo. Neste momento, o docente que aplicou a dinâmica saiu da sala para que os participantes pudessem responder livremente à pergunta estabelecida.

Para manter o sigilo da pesquisa, os sujeitos foram identificados como S1, S2, S3 até S22, sucessivamente. A identificação ocorreu de maneira aleatória, por sorteio e independente da ordem das entrevistas ou de quaisquer outros aspectos.

As folhas foram recolhidas e entregues a outro docente, para compilação das respostas e análise de conteúdo (AC) (Mozzato; Grzybovski, 2011), estratégia escolhida para análise dos dados. A AC compreendeu as seguintes etapas: organização dos dados, codificação, categorização, inferências e interpretação.

Segundo Bardin (2010), a AC tem como princípios a "exaustividade", "representatividade" e "pertinência". Deste modo, a categorização correspondeu a um roteiro semiestruturado elaborado de acordo com as respostas dos discentes à pergunta proposta. Os dados ainda foram analisados de acordo com o referencial teórico atual sobre metodologias ativas.

\section{Resultados}

Participaram da pesquisa 22 discentes, de ambos os sexos, com idade média de 23 anos. Na análise das respostas foram identificadas quatro categorias, sendo: a) quanto à estratégia utilizada como propulsora de revisão do conteúdo do módulo; b) eficiência da estratégia; c) construção do conhecimento adquirido e d) contexto de aplicação. Em cada uma das categorias separou-se alguns recortes das respostas que representavam a opinião dos discentes 


\section{a) Quanto à estratégia}

Nesta categoria observa-se que os sujeitos associaram a estratégia como uma intervenção dinâmica, em movimento, opondo-se ao modelo não participativo.

S4 "[...] é uma estratégia dinâmica."

S5 "[...] tornou a discussão mais dinâmica..."

$\mathbf{S 1 4}$ "[...] estratégia dinâmica e eficaz..."

S21 “[...] gostei muito da dinâmica..."

\section{b) Eficiência da estratégia}

Pode-se identificar que os discentes associaram a estratégia como uma forma adequada, consistente e significativa para a revisão do conteúdo.

S1 "[...] síntese de tudo o que foi visto no módulo, aguçando a memória"

S2 "[...] forma divertida de revisar o assunto..."

S3 “[...] estimula o raciocínio lógico através da recordação do tema estudado..."

S18 “[...] possibilitou a fixação do conteúdo trabalhado..."

S22 “[...] estratégia divertida e prática de buscar o conhecimento acumulado durante todo o módulo"

\section{c) Construção do conhecimento adquirido}

Os sujeitos participantes identificaram a estratégia como uma forma de se construir o aprendizado coletivamente.

S2 "[...] uma excelente atividade que envolve tanto o grupo, como os conhecimentos individuais..."

S3 “[...] promove a interação e o raciocínio coletivo..."

S4 "[...] utiliza de uma forma mais interativa e divertida" 
S21 "[...] estimulou o estudo individual e a discussão em grupo"

\section{d) Contexto de aplicação}

Os discentes associaram o conteúdo trabalhado à prática clínica, podendo assim significar sua aprendizagem de acordo com o contexto profissional exigido.

S6 "[...] através da recordação do tema estudado para assim dar uma hipótese diagnóstica do caso"

S7 “[...] os casos clínicos presentes no jogo evidenciou se o conteúdo já estava realmente compreendido..."

$\mathbf{S 1 2}$ “...] nos estimulou bastante a responder as perguntas e pensar nas possíveis possibilidades..."

$\mathbf{S 1 4}$ “[...] uma forma divertida e prática de buscar o conhecimento acumulado..."

$\mathbf{S 1 9}$ “[...] ajuda a relacioná-las com uma visão geral e raciocínio clínico”

\section{Discussão}

Diferentes são as possibilidades de cenários de aprendizagem em saúde, sendo que o ensino clínico, independente da área profissional, deve proporcionar ao estudante correlacionar os sinais e sintomas estudados com as manifestações do paciente. Assim, o discente poderá executar, posteriormente, a reflexão sobre a avaliação específica, o diagnóstico diferencial, o planejamento e a avaliação da própria intervenção, para acompanhamento de sua eficácia. Com esse objetivo e visando aumentar a motivação dos estudantes na modalidade problematizadora de ensino e aprendizagem, foi estruturada a dinâmica sob a forma de jogo educativo denominado como "Detetive de Linguagem".

Isso porque quando os jogos desenvolvem habilidades cognitivas importantes para a aprendizagem, envolvendo a resolução de problemas, a percepção, a criatividade, o raciocínio rápido, dentre outras habilidades, e caso tenham sido elaborados com o 
objetivo de atingir conteúdos específicos e para ser utilizado no âmbito acadêmico, são classificados como jogos didáticos (VOLANTE ZANON; SILVA GUERREIRO; OLIVEIRA, 2008), podendo, dessa forma, ser utilizados em diferentes populações e com objetivos diversos. Além disso, as autoras acrescentaram que a utilização de material didático-pedagógico inovador facilitou e aumentou a dinâmica do processo de ensino e aprendizagem. O mesmo foi descrito pelos estudantes participantes deste estudo, considerando a estratégia como dinâmica, divertida e que favorece a troca de conhecimentos.

O jogo favoreceu também a aquisição de conhecimento em ambiente de descontração, eliminando o temor do estudante quando seus conhecimentos são avaliados, como é possível verificar nos registros dos discursos apresentados neste estudo e no estudo de Barbosa et al. (2010). Ratifica-se também o exposto por Volante Zanon; Silva Guerreiro; Oliveira (2008), ao afirmarem que os jogos didáticos favorecem a motivação, o raciocínio, a argumentação e a interação entre os alunos e com o professor.

Outro aspecto importante do jogo didático é fazer com que a reflexão sobre determinado tema seja realizada, ajudando na fixação dos conceitos mais importantes, como o relatado pelos participantes deste estudo. Monteiro; Vargas; Rebello (2003), ao tratarem do tema doenças sexualmente transmissíveis e a síndrome da imunodeficiência adquirida (DST/AIDS) com 62 estudantes e 17 educadores, analisaram o "Jogo da Onda" sob diferentes perspectivas, constatando que apesar dos jogos didáticos facilitarem as ações educativas em saúde, poucos estudos se debruçam sobre suas repercussões, concluindo que vários fatores estão imbricados no jogo, como os aspectos culturais, o ambiente, a compreensão das regras, a coesão do grupo, entre outros.

Por outro lado, os jogos didáticos podem servir tanto no momento em que há a necessidade da apresentação de novos conceitos ou como fixação de conteúdos. A presente proposta de pesquisa foi o de apresentá-lo diante da necessidade de fixação de conteúdos complexos de um módulo problematizador no Ensino Superior em Saúde.

A fixação de conceitos complexos requer uma postura proativa do discente no ensino. Rieder; Brancher (2002), com a intenção de fixar conteúdos relacionados à matemática, desenvolveram software educacional para estudantes do Ensino Fundamental, de forma que a aprendizagem ocorresse de forma lúdica e motivadora, despertando o interesse dos estudantes no uso de novas tecnologias, levando-os a desenvolver linguagem, pensamento e concentração. 
Assim como observado por Patriarcha-Graciolli, Zanon, Souza (2008), o uso do jogo Detetive foi de grande aceitação entre os discentes do Curso de Fonoaudiologia, mostrando-se lúdico e eficaz como instrumento motivador da aprendizagem nos alunos.

Além disso, é importante considerar que a utilização de casos clínicos como parte do jogo pautam discussões para o raciocínio clínico em Saúde de forma indireta, já que o paciente não está diante do(s) avaliador(es). Sendo que, segundo Galdeano; Rossi; Zago (2003), o ideal para a construção de um caso clínico é a elaboração de um roteiro instrucional em que sejam discutidas as questões norteadoras do estudo (O que? Quem? Como? Por que? Qual?), apresentando-se os dados de identificação, a síntese dos problemas ou das alterações identificadas, com fundamentação teórica, as alternativas ou propostas do estudo, as ações implementadas ou recomendadas e discussão entre os envolvidos, ou, ainda, sobre a necessidade de discussão interdisciplinar, assim como realizado neste estudo. Cabe, no entanto, ressaltar, que para fins didáticos, os casos clínicos utilizados no ensino podem ser modificados para os objetivos desejados (YIN, 2015).

Assim consideramos que os jogos didáticos favorecem e facilitam a aprendizagem de temas complexos, tornando-a significativa, desenvolvendo habilidades, tanto no âmbito individual quanto coletivo, próximas daquelas que os universitários vivenciarão na esfera profissional, como preconizado por Campos; Bortoloto; Felício (2003). Além disso, as atividades lúdicas são mediadoras efetivas do processo de ensino e aprendizagem (COSCRATO; PINA; MELLO, 2010), como verificado neste estudo.

Entretanto, é importante enfatizar que apesar dos benefícios observados neste estudo na utilização de jogos didáticos na graduação em Fonoaudiologia são necessárias novas pesquisas, utilizando diferentes metodologias e com um número maior de estudantes, a fim de se verificar sua eficácia a longo prazo, bem como sua aplicação no Ensino Superior, vistos que os relatos sobre esse tema ainda são escassos.

\section{Conclusão}

As respostas obtidas evidenciaram a percepção positiva dos discentes quanto à utilização do jogo "Detetive da Linguagem" como método de ensino e aprendizagem, sendo que as principais percepções assinalaram que o jogo possibilitou a associação do conteúdo trabalhado à prática clínica, identificaram a estratégia como uma forma de se 
construir o aprendizado coletivamente, associando-a como uma intervenção dinâmica, consistente e significativa para a revisão do conteúdo.

\section{REFERÊNCIAS}

BARDIN, L. Análise de conteúdo. Lisboa: Edições 70, 2010.

BARBOSA, S. M.; DIAS, F. L. A.; PINHEIRO, A. K. B; PINHEIRO, P. N. D. C.; Vieira, N. F. C. Jogo educativo como estratégia de educação em saúde para adolescentes na prevenção às DST/AIDS. Revista Eletrônica de Enfermage, v. 12, n. 2, p. 337-41, 2010.

CAMPOS, L. M. L.; FELICIO, A. K. C.; BORTOLOTO, T. M. A Produção de Jogos Didáticos para o ensino de ciências e biologia: uma proposta para favorecer a aprendizagem. Cadernos dos Núcleos de Ensino, São Paulo, p. 35-48, 2003.

COSCRATO, G.; PINA, J. C.; MELLO, D. F. D. Utilização de atividades lúdicas na educação em saúde: uma revisão integrativa da literatura. Acta Paulista de Enfermagem, v. 23, n. 2, p: 257-63, 2010.

DORNELAS, R.; GUEDES-GRANZOTTI, R. B.; DOMENIS, D. R.; CESAR, C. P. H. A. R.; BARRETO, A. C. O.; SILVA, K.; IRINEU, R. A. Metodologias Ativas: Uma experiência na Fonoaudiologia. Distúrbios da Comunicação, v. 26, p. 815-821, 2014.

DORNELAS, R.; SILVA, K; CÉSAR, C. P. H. A. R.; OLIVEIRA-BARRETO A. C.; GUEDES-GRANZOTTI, R. B. Avaliação da Situação-Problema por Tutores e Discentes em um Curso de Graduação Vivenciado em Metodologias Ativas. Revista Ibero-Americana de Estudos em Educação, v. 11, n. 1, p. 245-248, 2016. DOI: 10.21723/riaee.2016.v11.n1.p245.

FONSECA, L. M. M.; SCOCHI C. G. S.; MELLO, D.F. Educação em saúde de puérperas em alojamento conjunto neonatal: aquisição de conhecimento mediado pelo uso de um jogo educativo. Revista Latino-americana de Enfermagem, v. 10, n. 2, p. 166-71, 2002.

GALDEANO L. E.; ROSSI L. A.; ZAGO M. M. F. Roteiro instrucional para a elaboração de um estudo de caso clínico. Revista Latino-Americana de Enfermagem, v. 1, n.3, p. 371-5, 2003.

GUEDES-GRANZOTTI, R. B.; SILVA, K.; CARMO, R. D.; DOMENIS, D. R. Metodologias Ativas e as Práticas de Ensino na Comunidade: sua importância na formação do Fonoaudiólogo. Distúrbios da Comunicação, v. 27, p. 369-374, 2015.

JUSTUS, J. F. C.; FRANCISCO, A. C. Detetive da saúde: a contribuição de um jogo de tabuleiro para o ensino e a aprendizagem na área da saúde. B. E. C. T., v. 5, n. 1, jan./abr. 2012. 
LEGEY A. P.; MOL A. C. A.; BARBOSA J. V.; COUTINHO C. M. L. M.

Desenvolvimento de jogos educativos como ferramenta didática: Um olhar voltado à formação de futuros docentes de ciências. Revista Educação em Ciência e Tecnologia, v. 5, n. 3, p. 49-82, 2012.

MOZZATO, A. R.; GRZYBOVSKI D. Análise de conteúdo como técnica de análise de dados qualitativos no campo da administração: potencial e desafios. RAC, v. 15, n. 4, p. 731-47, 2011.

MONTEIRO, S. S.; VARGAS, E. P.; REBELLO, S. M. Educação, prevenção e drogas: resultados e desdobramentos da avaliação de um jogo educativo. Educ. Soc., v. 24, n. 83, p. 659-78, 2003.

PATRIARCHA-GRACIOLLI, S. R.; ZANON, Â. M.; SOUZA, P. R. “Jogo Dos Predadores": uma proposta lúdica para favorecer a aprendizagem em ensino de Ciências e Educação Ambiental. Rev. Eletrônica Mestr. Educ. Ambient., v. 20, p. 2012-216, 2008.

RIEDER, R.; BRANCHER, J. D. Development of a micro world for the education of the Fundamental Mathematics, using OpenGL and Delphi. In: X Congreso Iberoamericano de Educación Superior em Computación en el marco de CLEI, 2002.

VOLANTE ZANON, D. A.; SILVA GUERREIRO, M. A.; OLIVEIRA, R. C. Jogo didático Ludo Químico para o ensino de nomenclatura dos compostos orgânicos: projeto, produção, aplicação e avaliação. Ciências \& Cognição, v. 13, n. 1, p. 72-81, 2008.

YIN, R. K. Estudo de caso: planejamento e métodos. Porto Alegre: Bookman, 2015 ZUANON, A. C. A.; DINIZ, R. H.; NASCIMENTO, L. H. Construção de jogos didáticos para o ensino de biologia: Um recurso para integração dos alunos à prática docente. R.E.C.T., v. 3, n. 3, p. 49-59, 2010.

\section{Como referenciar este artigo}

SILVA, Kelly da.; GUEDES-GRANZOTTI, Raphaela Barroso.; CÉSAR, Carla Patrícia Hernandez Alves Ribeiro.; DORNELAS, Rodrigo. O jogo como ferramenta de ensino e aprendizagem em fonoaudiologia. Revista Ibero-Americana de Estudos em Educação, Araraquara, v. 13, n. 1, p. 78-88, jan./mar. 2018. E-ISSN: 1982-5587.

Submetido em: 17/08/2016

Revisões requeridas: 10/12/2016

Aceito em: 02/07/2017 